\title{
A Hybrid System for Multimedia Conferencing on Android Technology
}

\author{
Nataasha Raul \\ Asst. Professor, \\ Sardar Patel Institute \\ of Technology, \\ Mumbai, India
}

\author{
Manas Churi \\ Student, \\ Sardar Patel Institute \\ of Technology, \\ Mumbai, India
}

\author{
Shruti Desai \\ Student, \\ Sardar Patel Institute \\ of Technology, \\ Mumbai, India
}

\author{
Neelam Gulrajani \\ Student, \\ Sardar Patel Institute \\ of Technology, \\ Mumbai, India
}

\begin{abstract}
Communication plays an important role in today's time. Globalization has led the need for crossover communication. Therefore multimedia system plays an important role in the overall communication between regions. Multimedia system provides seamless provision of multimedia services to its user. The conferencing system ensures that voice, video and other media forms are streamed efficiently to various individuals ensuring smooth real time communication. Currently, this multimedia system does not support this facility in all the mobile operating system. Thus we have proposed an Android based multimedia conference terminal system on $3 \mathrm{~g}$ mobile phones. In this way $3 \mathrm{G}$ users will be allowed to communicate with individuals across regions.
\end{abstract}

\section{General Terms}

Documentation, Performance, Design, Theory

\section{Keywords}

3G, Android, Client Server Architecture, Multimedia, Video Conference

\section{INTRODUCTION}

Cross region communication has become increasingly important due to globalization and trade. Multimedia conference system is put to use instead of traditional conference to enhance communication efficiency, save staff's time and reduce conference cost. The maturity of Internet application service and the wide spreading of broadband access technology help to accelerate the development of multimedia conference system. Due to the development in networks that is the emergence of $3 \mathrm{G}$ networks and introduction to new smart phones has led to the popularity of the conference system on mobile phones. 3G network provides high-speed data transmission capability. The new age smart phones are intelligent and sophisticated in features and hence support enormous applications. Due to the portability feature and personalization of trends in mobile terminals, applications like multimedia conference can be easily mounted on these terminals. At present, the mainstream mobile phone operating systems include Symbian, Windows Mobile, iOS and Android. The Android operating system is the newest among all these. It has $38.1 \%$ market share according to a survey in May 2011. This percentage has been increasing regularly with the rise of smart phone users. Under this background, we have designed and proposed a Android based multimedia conference terminal system on $3 \mathrm{G}$ mobile phone to facilitate $3 \mathrm{G}$ users to attend multimedia conference.
With the introduction of relatively low cost, high capacity broadband telecommunication services in the late 1990s, coupled with powerful mobile processors and video compression techniques, videoconferencing usage has made significant inroads in business, education, medicine and media. This type of communication allows long distance communication thus avoiding the need to travel to places to share ideas, views and also leading to reduction in global warming.

The rest of this paper is organized as follows. Section 2 describes related work done to get an insight of the technologies and architectures required for establishing a multimedia conference system. The proposed architecture of the multimedia conference system comprising of the building blocks of the system is presented in Section 3. In Section 4, the implementation of our application that is the working for to make a video conference call is described. Section 5 describes the evaluation of our system, where the design of our system and various options available to control or use the conference system is shown. The advantages of the proposed system over the existing system are given and are discussed in section 6. The various areas where are our system is applicable is described in Section 8. The conclusion is given in section 8 and it is followed by acknowledgement in Section 9

\section{RELATED WORK}

\subsection{Architecture of Multimedia Conference}

\section{System}

Figure 1 shows the topology architecture of the multimedia conference system. Here the typical kinds of users who can enjoy multimedia conference services are given. They are the telephone users, $3 \mathrm{G}$ cell phone users or the computer users. The network used for each of the user depends on the terminal type. If the terminal is a telephone then the network used is a switching system that is, PSTN. If the terminal is the computer then the network is the internet service and the networking device used is a router. For $3 \mathrm{G}$ mobile phones the network used is $3 \mathrm{G}$ and it has base stations which connect to the MSC. From the network, the communications through the gateway which is a network device mapping to all the OSI layers takes place. From there the conference system is managed and controlled by the Conference control server and media server. These two servers help in establishing a conference as well as managing all the details of conference call. 


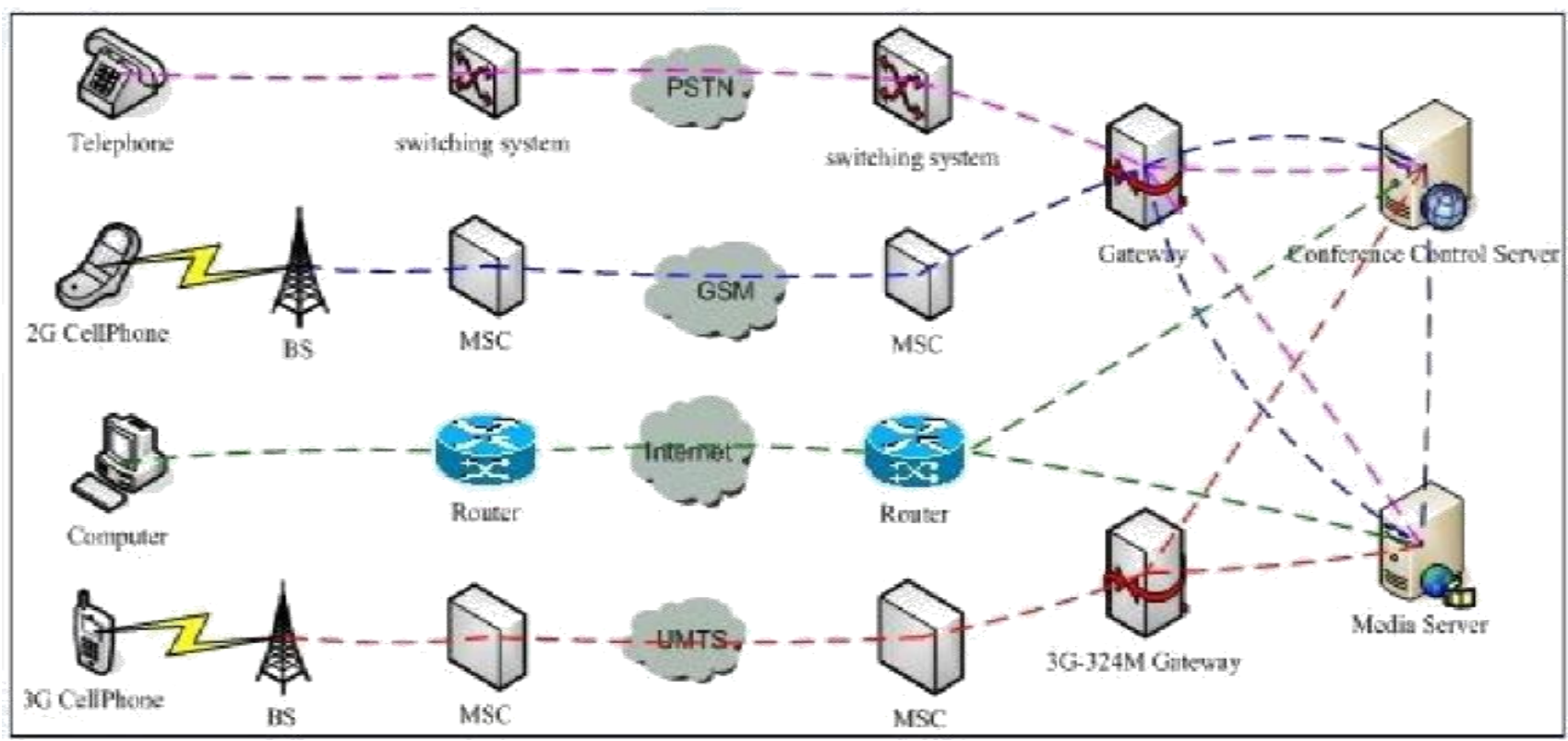

Fig 1: Topology Architecture of Multimedia Conference System ${ }^{[3]}$

The paper "The Design and Implementation of Multimedia Conference Mobile Terminal Application" describes module based architecture. It adopts separate logic control and media module. It bases on public network like PSTN, 3G, GSM and Internet. It provides multi-party conference services for enterprise users and general users. It can hold several conferences concurrently.

It allows multiple types of terminals access the multimedia conference server and supports switching between them. From this paper we have studied that various modules are formed in order to perform the corresponding task. Like the 'Multi-task concurrent execution subsystem' allows multiple task to be carried on while the conference is on and will not hinder front camera video capture. Similarly "View conference information' will give details about the ongoing conference. 'Switch terminal will help the user to be or go out from the main video view. 'Participation and Certification speech' gives an overview of the conference details like users who had participated and confirmation of the same. 'Conference Service Manager Subsystem' gives the various services regarding the conference. The terminals specified are the videoconferencing equipment. They are connected through router, switch, base stations, node depending on technology processing module are separated from each other.

This architecture helps us to understand the basic module processing like the media server which helps in media management for the conference.' User and group management' deals with the managing of group of users who participated in the conference. ${ }^{[2]}$

Due to the emergence of wireless communication, free calls can be made all around the world using the latest terminal system and networks. Wi-Fi is constraint on area basis but $3 \mathrm{G}$ network is present even in remote regions these days. A 3G network enables its users to make cheap calls or free calls anywhere in the world. The only requirement is the presence of the application on the smart phone and installing it to enjoy the application service.

H.323 is a protocol used for video conferencing. H.323 specifies system components, control messages, and procedures that define communications among H.323 components. ${ }^{[5]}$ Later on, SIP (Session Initiation Protocol) emerged as a efficient video conferencing protocol. As an extensible signaling protocol, SIP (session initiation protocol) can be applied in developing video conference system. [6]

The study of the android OS, 3G technology and the multimedia conference architecture helped us to propose our own multimedia conference system given in section 3 .

\section{PROPOSED ARCHITECTURE OF MULTIMEDIA CONFERENCING SYSTEM}

The conference control system module includes authentication server which contains the user database and the gatekeeper which keeps a database of IP

Gatekeeper:-Gatekeepers are also called Session Border Controllers. They are used to proxy H.323 calls by combining them with gateway functions. A gatekeeper monitors simultaneous requests for connection to prevent network congestion. It acts as a central administrative entity while controlling the interactions of a zone which also includes terminals and multipoint control units. The gatekeeper performs various functions like authentication, authorization, private branch exchange (PBX) and telephone directory services, call control and routing. Other functions may include monitoring the network for load balancing and real-time network management applications, intrusion detection and prevention, and providing interfaces to legacy systems

Authentication Server: - This kind of server will verify whether the users trying to use the system are authorized users. As a result the authorization of user is done by this server and a user gets a chance to use this system if her/his authority is proved. So, it acts as a entry point for the users to use

system 


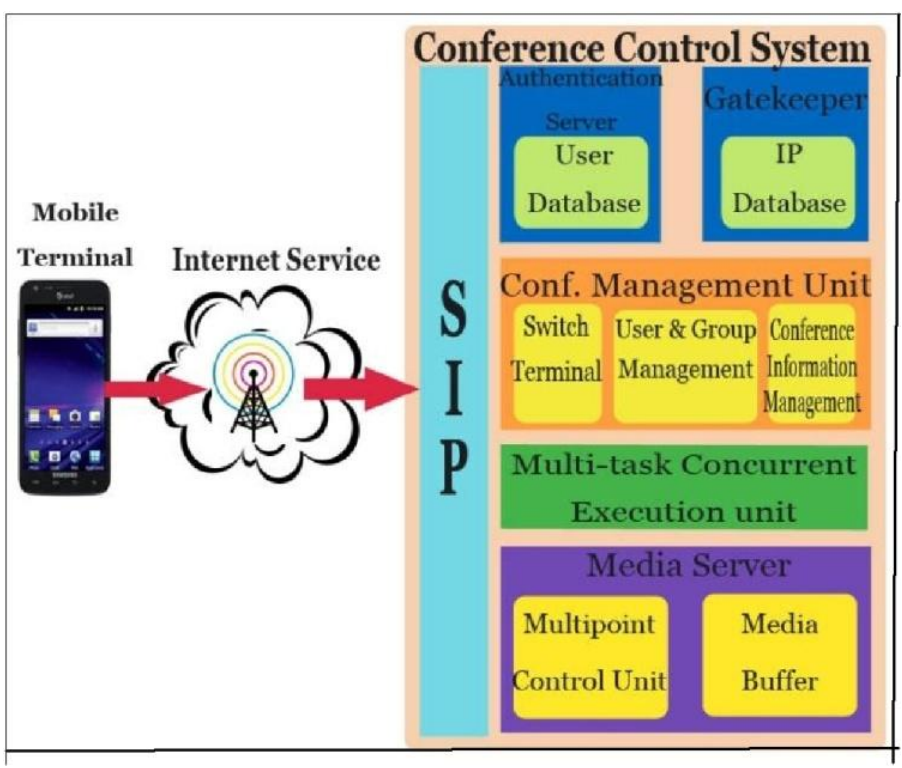

Fig 2: Multimedia Conference System Architecture

User database: - This database is used to maintain records of all the users who have already registered to the system.

IP Database:-IP database is used to store the IP addresses of mobile terminal systems. The gatekeeper will help in getting the IP address of the mobile terminals. These IP addresses are stored in order to communicate with mobile terminals.

The conference management unit includes the switch terminal block, user and group management block and the conference information management block.

Switch Terminals: - This ensures that other user comes into focus in conference call when there is a switch over to next conference candidate.

User and group management: - It deals with the managing of group of users who participated in the conference.

Conference Information management: - This deals with the job of keeping track of the conference information and managing it well for further purposes. Conference information may include the number of candidates in a conference, the duration of the conference and time and date of the same conference.

Multi-task Concurrent execution unit: - This unit ensures that the users are free to perform multitasking when a conference is going on. The unit makes sure that the user can switch between other functions of the smart phone and is not constraint to the conference application during a conference call.

The media server module includes Multipoint Control and Media Buffer:

Multipoint Control Units:- It consists of multipoint controller (MC) and multipoint processor(MP). It is used for controlling multipoint accesses. As in video conferencing, multipoint conference stations will be there according to the no of users in the conference, this managing unit is used to control all these access points.

Media Buffer:-Media buffer is used to store the videos streamed by the mobile users. These videos which are streamed are the ones which will be captured by the front camera of mobile terminals. These videos need to be stored temporarily. Hence media buffers are used.

Terminals:- These are the fundamental elements in any network. They are basically the equipment which the users get hold of in order to enjoy a particular service. This service needs to be utilized using this equipment. A terminal can be a cell phone, computer, telephone. The service should be compatible on the device used by the user.

SIP: -SIP stands for session initiation protocol. It is an application layer control (signaling) protocol for creating, modifying, and terminating sessions with one or more participants. These sessions include Internet telephone calls, multimedia distribution, and multimedia conferences. ${ }^{[6]}$

Internet Service: - Internet service here is the 3G network. The $3 \mathrm{G}$ network is the third generation of mobile networking and telecommunications. The $3 \mathrm{G}$ network has eliminated the limitations of $2 \mathrm{G}$ network and has included many new features. The transfer rate of $3 \mathrm{G}$ enables fast transmission of all forms of media. Due to this, forms like voice and videos can be easily streamed without any delays or jitters.

\section{IMPLEMENTATION OF MULTIMEDIA CONFERENCING SYSTEM}

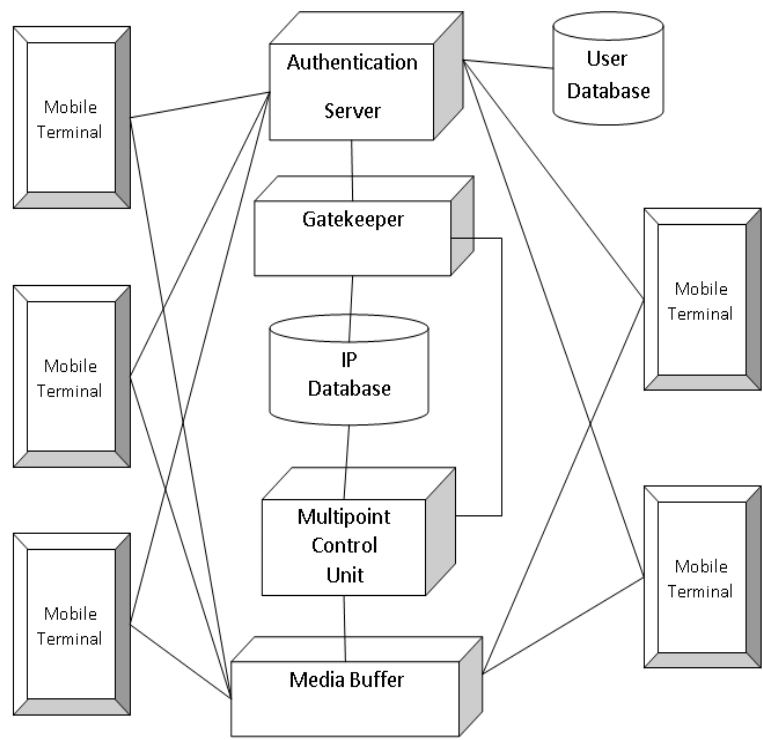

Fig 3: Functional architecture of conference system.

The specific process is described as follows

- Initially the application has to be installed on cell phone with android platform.

- Then user has to authenticate itself with its username and password with the authentication server.

- The authentication server has the user details in the user database which helps in authenticating the user.

- The authentication server will also get the IP address of the mobile terminal at the same time.

- The gatekeeper does the job of getting the IP address and will store in the IP database.

- Multipoint Control Units utilizes controllers to support multipoint conferences. 
- The front camera will help in capturing the video of the user.

- The application implementation involves running of a thread where in the front camera is capturing the video.

- There is a second thread which is doing the job of streaming the video and storing it temporarily in media buffer

- The multipoint control unit which handles this media buffer streams the video information to the user in the conference.

- Similarly the same mechanism will work on all the user mobile terminals.

The main view will allow the user to either sign in, sign up to register or help view to get the help for using the application. The details about the application will be given in the about view. From the main view the user can edit conference, delete conference or attend conference. The delete conference ends at the conference view. After attend conference user can either switch terminal, terminate call or participate in the call. The call is a video / voice call. Edit conference will lead the user to conference edit view. In this way transition from one state to another is shown in Fig 4.

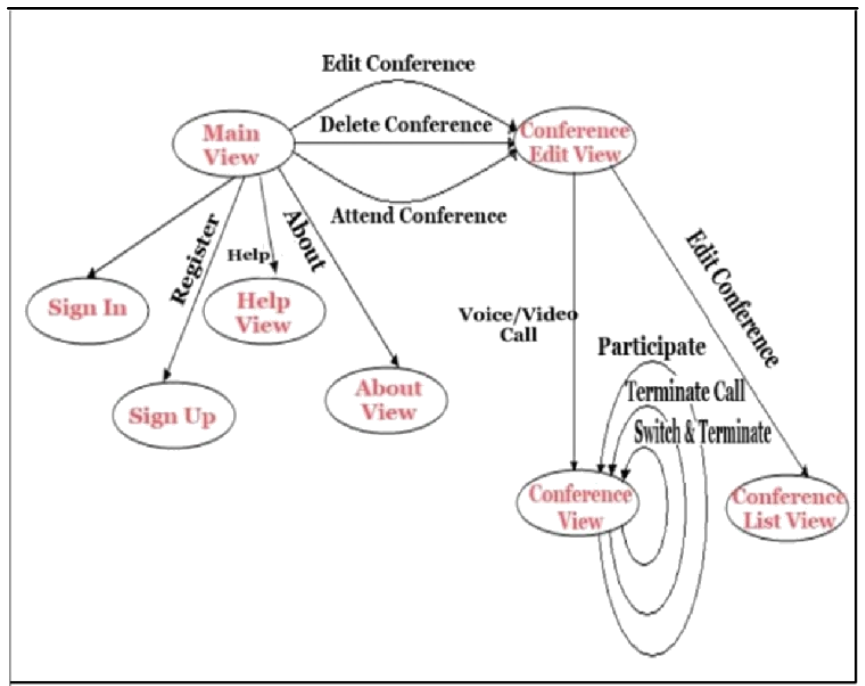

Fig 4: Android view switch of Conference System

\section{EVALUATION}

We have installed the multimedia conference mobile terminal application on Android OS phone Samsung Galaxy note and done the experiment of actively attending conference to evaluate the functions of the application.

The application snapshot is shown in fig 5 . The video view will show the video captured by front camera of the user to who call was made. Also other users who are not active but are in the conference can be seen. That is called as the switch terminal. Also if someone wishes to add a person on conference then the number needs to be entered in order to make the call. The person can also end the conference in a midst of an ongoing conference.

Figure 6 shows the main view of the multimedia conference system. The user can view conference which shows the details of the conference being held. Attend Conference helps the user to become a part of the ongoing conference. Edit Conference helps the user to edit the conference information which was previously held. Delete Conference helps the user to remove the details of an already occurred conference. Thus the main view helps the user to save information of an already held conference, or edit it or delete it.

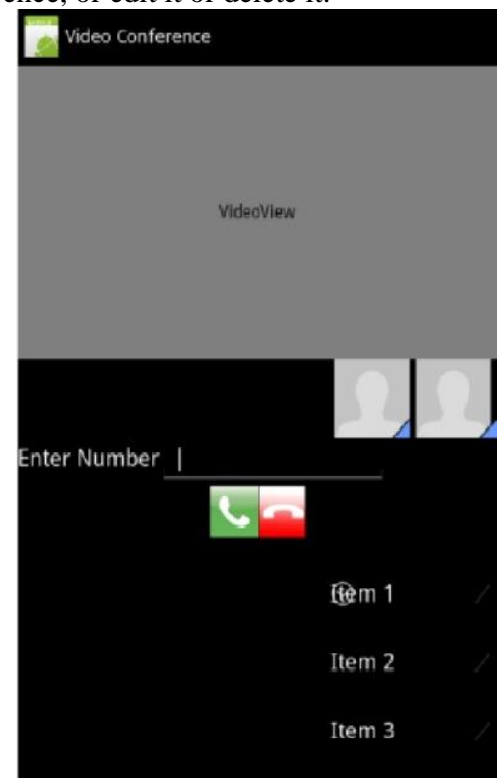

Fig 5: Application Snapshot of Chat Window

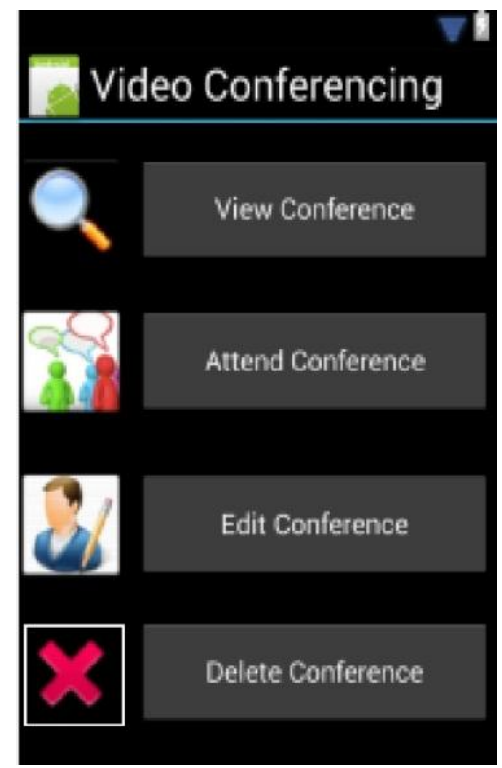

Fig 6: Main view of Multimedia Conference Application

\section{ADVANTAGES OF THE PROPOSED SYSTEM OVER THE EXISTING ARCHITECTURE}

- The presence of media buffer ensures that even if there is hardware limitations regarding memory in the cal lee's phone the conferencing will be done efficiently. This is because the media buffer stores the streamed video temporarily and stream it sequentially instead of sending bulk multimedia

- In case of mobile devices IP allocation is dynamic so setting up communication is difficult. The dynamic retrieval of the IP of the mobile terminals helps in communicating with the terminal without any IP search hindrances.

- Android supports powerful and a highly efficient hardware and has facilities for various multimedia 
applications, playing audio and video files. It not only supports a number of media players but also all kinds of audio/video formats, HDMI connectivity and DLNA video streaming. On the other hand, Symbian phones have a default media player, thus not supporting different formats and they also lack DLNA connectivity.

\section{APPLICATIONS}

Video Conferencing has its applications in a wide range of areas. Some of its applications are:

\section{Academic help}

Videoconferencing provides students with the opportunity to learn by participating in two-way communication forums. Furthermore, teachers and lecturers worldwide can be brought to remote or otherwise isolated educational facilities. Students from diverse communities and backgrounds can come together to learn about one another, although language barriers will continue to persist.

\section{Interactive communication in office environment}

Videoconferencing can enable individuals in distant locations to participate in meetings on short notice, with time and money savings.

\section{E-learning}

Faculty members or teachers can keep in touch with classes while attending conferences. Guest lecturers can be brought in classes from other institutions. Researchers can collaborate with colleagues at other institutions on a regular basis without loss of time due to travel. Schools with multiple campuses can collaborate and share professors. Student can interview with employers in other cities

\section{Medical assistance}

Videoconferencing is a highly useful technology for real-time telemedicine and tele-nursing applications, such as diagnosis, consulting, transmission of medical images, etc... With videoconferencing, patients may contact nurses and physicians in emergency or routine situations; physicians and other paramedical professionals can discuss cases across large distances.

\section{CONCLUSION}

The networking field is expanding on a great scale incorporating many new technologies and applications. This network with immense advancements has provided many entry points for smooth communication. Voice, video and document sharing has become a mundane task due to the emergence of advanced technology. Multimedia conference system is becoming increasingly popular among enterprises and government departments by right of its efficiency and cost-saving features. In this paper, we have designed and implemented a Android based multimedia conference mobile terminal application, with which $3 \mathrm{G}$ mobile phone users can attend multimedia conference anytime and anywhere and enjoy high-quality, real time and smooth conference experience.

\section{ACKNOWLEDGEMENT}

Mr. Manas Churi, Ms. Neelam Gulrajani and Ms. Shruti Desai would like to thank Prof Mrs. Nataasha Raul for her constant encouragement and help. Her guidance in understanding the various aspects related to this project was vital.

\section{REFERENCES}

[1]. Fatna Belqasmi, Chunyan Fu, Mohammed Alrubaye, Ericsson Canada Roch Glitho, Ericsson Canada and Concordia University, Design and Implementation of Advanced Multimedia Conferencing Applications in the 3GPP IP Multimedia

\section{Subsystem}

[2]. Ha Jingjing, Shang Yanlei, Chen Junliang, Tan Gang

The Design and Implementation of Multimedia Conference Mobile Terminal Application

[3]. Li Shangmeng, Shang Yanlei, Ha Jingjing, Chen Junliang The Design and Implementation of Multimedia Conference Terminal System on 3G Mobile Phone

[4]. G. Camarillo and M.-A. Garcia-Martin , Wiley,2004, The 3G IP Multimedia Subsystem: Merging the Internet and the Cellular Worlds

[5]. Wanjiun Liao, Member, IEEE, Mobile Internet Telephony: Mobile Extension to H.323

[6]. Zhen Yang; Huadong Ma; Ji Zhang; Sch. of Comput. Sci. \& Technol., Beijing Univ. of Posts \& Telecommun., China, A dynamic scalable service model for SIP based video conference

[7]. Van Staalduinen, K.; Trommeien, P.H., Standards for third generation mobile communication

[8]. Li Hao, Zhang Gang, Liu Tao, Research on Video Conferencing Based on Hierarchical Ad-Hoc Network

Zhang Yanyan; Yao Yuan, SIP- based multimedia conference system design and implementation

[9]. Junchao Li; Weimin Lei; Xiuwu Zhang, Design and implementation of SIP-based centralized multimedia conferencing system

[10]. Akkanen, J.; Karonen, O.; Porio, J., Peer to Peer Video Streaming on Mobile Phones

[11]. Farkas, L.; Aczel, K., Streaming videos from smart phones: a feasible study 